\title{
Simplified approach to determine the requirements of a "flexible nuclear reactor" in power system with high insertion of variable renewable energy sources
}

\author{
Anne-Laure Mazauric ${ }^{1, *}$, Pierre Sciora ${ }^{1}$, Vincent Pascal $^{1}$, Jean-Baptiste Droin ${ }^{1}$, Yvon Bésanger ${ }^{2, * *}$, \\ Nouredine Hadjsaïd ${ }^{2,3, * *}$, Quoc Tuan $\operatorname{Tran}^{4}$, and Nicolas Guyonneau ${ }^{1}$ \\ ${ }^{1}$ CEA, DES, IRESNE, DER, F-13108, Saint Paul lez Durance, France \\ ${ }^{2}$ Univ. Grenoble Alpes, CNRS, Grenoble INP, G2Elab, 38000 Grenoble, France \\ ${ }^{3}$ Energy Research Institute at NTU, Nanyang Technological University, Singapore \\ ${ }^{4}$ CEA, LITEN, F-73375 Le Bourget-du-Lac, France
}

Received: 8 March 2021 / Received in final form: 30 September 2021 / Accepted: 24 November 2021

\begin{abstract}
The objective of the paper is to study the potential behaviour of a power system with high share of nuclear and less thermal plants, in which variable RES insertion increases - for example the French case -, in order to determine the specifications for the design of a potential nuclear reactor with high "manoeuvrability". Moreover, the flexible reactor may participate more in the supply - demand balance and in particular during large frequency fluctuations caused by the high variability of RES. The studies are carried out with the PowerFactory software, which make it possible to highlight specific needs regarding the power ramp for an "ideal" flexible nuclear reactor. Using a benchmark network, the Kundur "2 areas-4 machines", the flexibility requirements are obtained as a function of the grid disturbances. For this purpose, the penetration of variable RES is progressively increased, while nuclear power is reduced and thermal power plants are totally suppressed. The study shows that a drop in RES production directly impacts the minimal frequency. A faster response speed of nuclear power makes it possible to restore this stability and return to normal operating conditions imposed by the grid operator. This paper describes therefore the process of obtaining the flexibility criterion for different cases of insertion of variable RES.
\end{abstract}

\section{Introduction}

\subsection{Context}

The balance between electricity production and consumption must be ensured, at all times, on an electric power system. The large-scale penetration of variable Renewable Energy Sources (RES) such as wind and photovoltaic generation sources, which are not "dispatchable" in the electric mix, represents a challenge for the fulfil of this supply-demand balance. The compensation of the production/consumption fluctuation is currently ensured by using controllable generation units. These units are mainly based on traditional fossil fuel (coal, gas, etc.) and hydraulic (hydroelectric barrage or pumping/turbining).

However, to achieve goals regarding $\mathrm{CO}_{2}$ emissions, thermal energy plants should be replaced by low-carbon energy units. While, as stated above, some low-carbon

\footnotetext{
* e-mail: anne-laure.mazauric@cea.fr

** Institute of Engineering Univ. Grenoble Alpes
}

energy sources may lead to instabilities in the grid. For example, in 2018, variable RES represented 18\% [1] of the French installed capacity, whereas the French law and energetic scenarios plan that their share should increase within a decade to represent more than $50 \%$ of the French installed capacity $[2,3]$. In order to meet the $\mathrm{CO}_{2}$ reduction targets, low-carbon flexible levers are necessary. Indeed, conventional thermal plants will be progressively removed in the next decade in order to fulfil the assigned target, while today, they are highly used by transmission system operator (TSO) to control power system stability. Flexible solutions such as power modulation of generation sources to accommodate electricity demand, storage or even load shedding are low carbon ways of adapting electricity production to consumption. They participate in the supply-demand balance, which is a necessary condition for the proper operation of a power system from the stability point of view. Should this balance not be reached, the frequency differs from its nominal point, $50 \mathrm{~Hz}$, which in the worst case may lead to blackout. However, the paper will focus on the study of flexibility of nuclear power plants, 
thus non-nuclear potential levers such as storage, demand management or cogeneration are out of the scope of this study. The French case is taken as an example of the approach, since the French electrical system is strongly nuclear and aims to insert more variable RES.

Nuclear power plants (NPP), which are low carbon emitters, contribute also, but in a lesser extent than conventional thermal or hydraulic power plants, to the balance and frequency control thanks to their "load-following" mode. Nevertheless, their internal operating constraints prevent each nuclear power plant from participating strongly in stability control due mainly to pellet-to-clad interaction [4]. The nuclear flexibility solutions found in the literature [5-8] focus mostly on the downstream part of the nuclear reactor, by using mechanisms that are located outside the turboalternator group in the energy transmission chain. On the contrary, the innovative idea of this study is based on improving nuclear manoeuvrability, looking for flexibility requirements that could then be addressed by the nuclear power plant design in other studies.

\subsection{Approach and contributions}

The purpose of this paper is to give a first overview of the requirements that flexible reactors will have to achieve, in order to accommodate future power system disturbances due to the increase of variable RES and phasing out of thermal power plants. This paper describes a methodology, applied to the French case, aiming at identifying specifications for the design of an ideal flexible nuclear model that ensure power system stability with an increasing share of RES in the electric mix. In other words, the paper suggests an original approach leading to recommendations for a future design of a new nuclear model, which will be more suitable for participating actively to the supply and demand balance from power system perspectives with high RES proportion. The study only takes into account nuclear power as a vector of flexibility.

The sections of the paper reflect the main steps of the methodology. Indeed, the study encompasses the following sections:

- Section 2 describes the main hypotheses that are suitable for carrying out the approach.

- Section 3 describes the network and the different elements of the power grid. For example, it requires the adaptation of existing model; a specific nuclear regulation and variable RES productions model in order to simulate an equivalent power system to the French grid.

- Section 4 gives the different steps for gradually increasing variable RES production and decreasing thermal resources in order to define flexible specifications for nuclear electric power in different mix cases.

- Section 5 deduces how flexible nuclear power plants can improve the stability of the system with a high proportion of variable RES, before the conclusion in the last section.

\section{Scope of the study}

This part explains the framework of the study, i.e. the main hypothesis required to define the nuclear flexibility specifications. The complex context of electrical networks requires choices to be made regarding the modelling of network components and the spatial and temporal fields of study, namely:

- the type of network of interest.

- the type of disturbances taken into account.

- the time scale of the simulations, in other words, the speed of the system to respond to a disturbance.

- normal operation.

French details are presented in the following for information. Then, the studied case is supposed is included in this scope.

\subsection{Transmission network}

Each current French nuclear reactor can generate a high power (about $1 \mathrm{GW}$ ) and is connected to the transmission grid. The electrical system used for the study is therefore a high-voltage transmission network connected to loads modelling distribution networks and consumer sites. In addition, it is possible to connect variable generation farm directly to a high-voltage transmission grid if the farm capacity is large enough ( $>12 \mathrm{MW}$ by connected farm), considering that the choice of the voltage transmission level for the connection of a power plants depends on its maximum power output.

\subsection{Power grid disturbances}

An imbalance between generation and consumption can lead to instabilities in the power grid. This imbalance can be caused by various power grid disturbances such as meteorological hazards (lightning, storms, frost, extreme cold, heat waves), failure of network components (shortcircuits, equipment failure, maintenance of critical components, etc.) or load variations (changes in consumption behaviour depending on the time of day). In this paper, only load variations and fluctuation of variable RES productions are taken into account (as an example of disturbance). RES disturbance is described in Section 3. Load variation and RES fall are therefore the events triggering the transients studied in the subsequent sections. Nevertheless, the Transmission System Operator (TSO) must ensure stability at all time, meaning maintaining pertinent power grid variables (frequency and voltage) within their operating range thanks to the various levers as shown in Table 1. For example, hydraulic storage, demand response, frequency control, or more recently coupling of several energy systems (cogeneration, hybrid systems) are deployed for that purpose.

\subsection{Primary frequency control}

Frequency control is directly related to the power supplied by the primary resource (nuclear thermal power is converted into mechanical energy at the turbine level) according to the equation hereafter, which is derived from the fundamental principle of dynamics applied to rotating shafts.

$$
J \frac{d \Omega}{\mathrm{d} t}=\sum C_{\text {mechanical }}-\sum C_{\text {electrical }}
$$


Table 1. Frequency control time-scale comparison [9].

\begin{tabular}{llll}
\hline & Primary control & Secondary control & Tertiary control \\
\hline Time scale & $<30$ seconds & 30 seconds to 15 minutes & 15 to 30 minutes \\
\hline
\end{tabular}

with $J$ the moment of inertia of the turbine-generator unit in $\mathrm{kg} / \mathrm{m}^{2} ; \Omega$ the rotation speed of the generator (in $r p m$ or $\mathrm{rad} / \mathrm{s}$ ) which represents an image of the grid frequency because; $\Omega=\frac{2 \pi f}{p}$ where $f$ is the grid frequency and $p$ the number of pole pairs of the generator; $C_{\text {mechanical the torque }}$ (in N.m) of the mechanical power supplied by the turbine via the rotor; $C_{\text {electrical }}$ the torque (in N.m) imposed by the power grid through the generator, it represents the "resistance" of the power absorbed by the consumers (in $\mathrm{Hz}$ ).

As an assumption, the study is limited to participation in the frequency control of NPPs. The other controls, voltage for example, are set but their influence is considered not to be significant in our study, as such controls are transparent with respect to the primary and secondary circuits of a nuclear reactor.

In order to keep this balance and ensure power grid stability, a hierarchical frequency control consisting in three successive controls is designed to act on the power output in order to restore the nominal frequency of $50 \mathrm{~Hz}$, to adjust contractualized power flows on interconnection lines and to reconstitute the needed power reserves.

The primary control restores quickly (less than 30 seconds) the generation-consumption balance with the objective of bringing the frequency at a value close to (but different from) the rated frequency. The primary control is automatic and is implemented as a local control loop (called speed governor) on each generator, which operates on the inlet valve of the turbine. In case of a change in the electrical set point, the turbine inlet flow rate is regulated in such a way that the power produced by the turbine varies. It means that this control is decentralized and the production system needs a permanent and sufficient reserve of power to operate correctly. In case of strong disturbances, the control rods can be adjusted in the framework of the primary control.

The secondary control is also automatic but centralized and located at the main French dispatching centre. It is used to bring back the frequency to the nominal operating point $50 \mathrm{~Hz}$ in less than $15 \mathrm{~min}$, and to adjust contractualized power flows on interconnection lines. It acts by modifying the reference signal of primary control loops of some qualified generators [10].

The tertiary control is manual and located at the main French dispatching centre. It finds the most efficient operating point from a technical and economic point of view in a longer period $(<30 \mathrm{~min})$ with a re-dispatching of power productions while reconstituting operating power reserve of secondary control [10].

Moreover, the impact of intermittent energy variability (solar and wind), as well as natural grid disturbances, may occur on short time scales of seconds to minutes. As wind turbines and solar panels are intermittent, they are by nature variable and dependent on seasonal, weekly, daily and permanent climatic conditions, which can sometimes introduce a strong imbalance between power generation and consumption. In the event of clouds for solar panels, and strong gusts or wind fall for wind farms, their generation level can drop sharply in a few seconds while consumption remains unchanged. These types of generation are not dispatchable, the frequency control is then mainly managed by the other conventional generating units such as thermal, hydraulic or nuclear groups, but researches are currently conducted to integrate RES at least in the primary frequency control mechanism.

Nowadays, thermal (gas and diesel) and hydraulic power plants participate more proportionally per unit than NPPs in primary control due to their short response time. Indeed, current nuclear power at the scale of its fleet (63 GW nuclear installed capacity in 2018 in France [1]) is quite capable of modulating its power rate in load following. Nevertheless, the quick power modulation capacities of the reactor itself are more limited (maximal power ramp of $5 \% \mathrm{Pn} / \mathrm{min}$, where $\mathrm{Pn}$ is the rated output power), mainly due to safety criteria $[4,11]$. In comparison, as shown in Table 2, the start-up times and ramp-up capacities of the other standard production sources (hydraulic, gas, diesel or coal) are much higher than nuclear. Therefore, in response to rapid disruptions $(<30 \mathrm{~s})$, the use of conventional hydraulic or thermal power generation units (gas, diesel) is prioritized.

The impact of the RES variability (solar and wind), as well as load fluctuations, occur on short time scales of a few seconds to few minutes. That is the purpose of the primary control to restore the generation/consumption balance in less than 30 seconds, as stated before (Tab. 1). Therefore, only primary frequency control is considered in the subsequent sections.

\subsection{Normal operation}

The transients simulated in this study occur during normal operation of the network and when the nuclear power plant is fully operational for load-following. This means that the primary control reserve is sufficient to respond to a power grid disturbance. For example, 2.5\% of reactor nominal power is typically kept for primary frequency control [13].

A penalizing event but characteristic of normal operation requires that the frequency remains in the acceptable range around $50 \mathrm{~Hz}$, i.e. $\pm 0.2 \mathrm{~Hz}$ during normal operation and $\pm 0.8 \mathrm{~Hz}$ transiently [14]. 
Table 2. Flexible capabilities of conventional plants [12].

\begin{tabular}{ll}
\hline & Approximate maximal power ramp \\
\hline Hydraulic & 0 to $100 \% \mathrm{Pn}$ in less than 1 minute \\
Diesel & $25 \% \mathrm{Pn} / \mathrm{min}$ \\
Combined cycle gas & $7 \% \mathrm{Pn} / \mathrm{min}$ \\
Gas turbine & $20 \% \mathrm{Pn} / \mathrm{min}$ \\
Coal & $5 \% \mathrm{Pn} / \mathrm{min}$ \\
Nuclear & $5 \% \mathrm{Pn} / \mathrm{min}[4]$ \\
\hline
\end{tabular}

\section{Modelling of the power system}

\subsection{Simulation tool for the power system dynamics}

Models and simulations used for the study are based on the power system dynamic simulation software PowerFactory developed by DIgSilent [11]. This software provides the correct time scale (30 s for primary control), as described in Section 2.3 and involves relevant physical phenomena, i.e. electromechanical transients due to frequency control. Many models of network elements such as synchronous machines and speed governors are available in its library.

The software has a module on RMS (Root Mean Square) analysis, adapted for the simulation of electromechanical disturbances, and for the calculation of power flows (with, for example, distribution of power production by unit in steady-state). PowerFactory takes advantage of the mathematical methods of variable step integration to simulate both stability phenomena and slow dynamics of electrical systems, achieving the optimal trade-off between accuracy and calculation time. It is thus possible to analyse over several hours the interaction of fast and slow settings or the succession of slow evolutions linked to consumption variations and sudden incidents such as short-circuits or unit tripping.

\subsection{Description of the electric network}

The chosen method used to identify the flexibility requirement, which is the objective of this methodology approach, is to model power transients on a high-voltage network and to observe the frequency evolution during normal operations. To do this, the study focuses on an electric network that is as simple as possible and which verifies the hypotheses stated in Section 2.3. Indeed, the detailed modelling of the French power system is complex, and is not achievable in the framework of this study. The precise and accurate network data are confidential and are property of the TSO. A simple benchmark network is then chosen and some modifications are made in order to be as representative as possible of the power system presented in the previous section about the scope.
3.2.1 Kundur's electric network and additional static generators

The so-called "2-area 4-machine" network of Kundur [15] is used to develop the approach and simulate power transients. This network has the advantage of being simple, of having fully opened data, and of having been widely benchmarked in the literature of the power system scientific community $[16,17]$.

In details, the Kundur's electric network, shown on Figure 2, is a high-voltage transmission system divided into two areas ( $\mathrm{A}$ and $\mathrm{B}$ ) linked by a corridor of 2 lines in parallel. Each area includes two synchronous generator units of 900 MVA maximal power and are connected to a load of respectively $967 \mathrm{MW}$ and $1767 \mathrm{MW}$ modelled by a constant power load. The machines in zone A (left) and the machines in zone B (right) differ in the inertia of the alternator. Maximal active power is $810 \mathrm{MW}$ for all generators. The lines and transformers are identical in both areas. Corridor lines are more than $200 \mathrm{~km}$ long. The four machines may represent classical power units such as nuclear, thermal or hydraulic power plants.

An equivalent static generator is added to the original network, meaning that the generator has no rotating machine and may represent a variable RES unit. This generator is connected to a load bus through a step-up transformer, as large RES farms are generally not located nearby traditional power plants.

\subsubsection{Initial power repartition conditions of the load flow computation}

The reference network of this study, adapted from Kundur's network, is considered in the following as an equivalent of the current French electric energy mix including variable renewable energies production, the latter being represented by a static generator. The initial distribution of loads and generating sources is specified in Table 3. Two out of four machines are of nuclear type because of the high proportion of nuclear in the French mix ( $\sim 50 \%$ of the French total power capacity).

The original "4-machine" network was initially designed to model low frequency inter-area and intra-area power oscillations. This is the reason why as soon as the load flow 


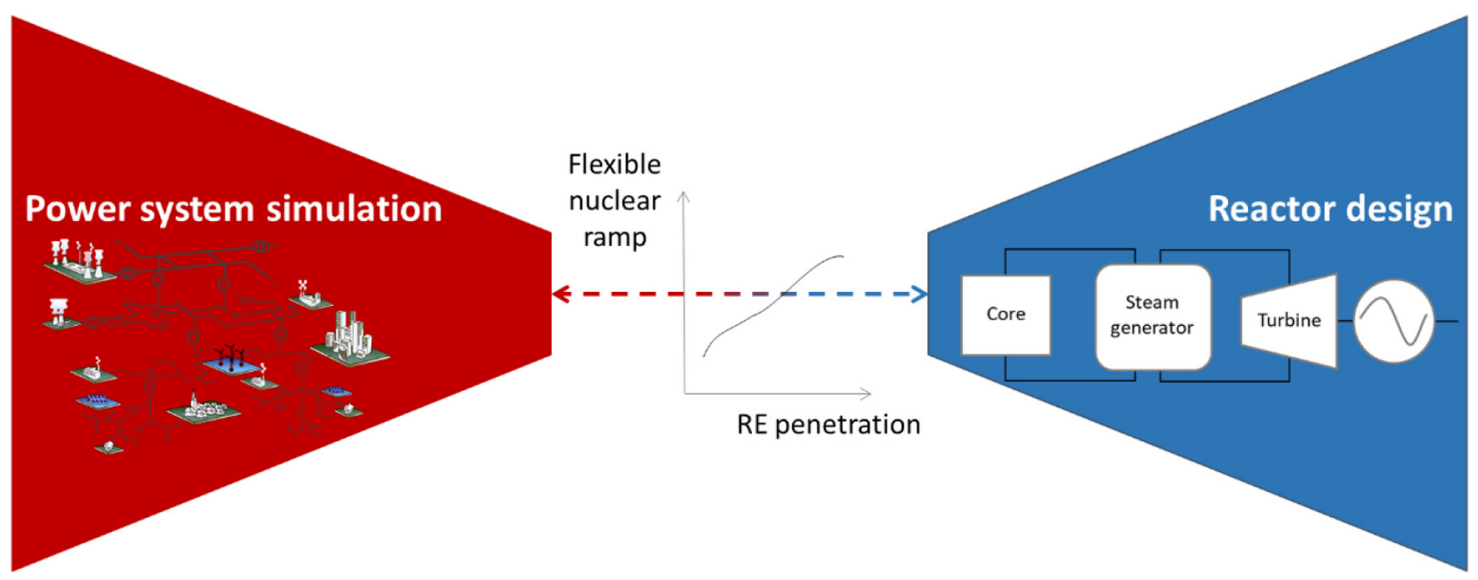

Fig. 1. Scheme of the global approach.

deviates from the nominal operating point (active power allocated to each components of the grid) described in the table below, frequency instabilities are quickly observed. Thus, the simulations of the study perform close to the initial operating point; power distribution between the four machines is then limited.

Figure 3 shows two examples of transient simulated with PowerFactory. Both transients show a frequency fall due to a deviation in power of $54 \mathrm{MW}$ either due to the loss of production or to an increase of the load. In both cases, the system returns to a stable state in the correct operating range (close to $50 \mathrm{~Hz}$ ) and in a relatively short time thanks to primary frequency control. The system behaviour is similar in both cases; it means that the frequency dip is the same with a production fall or a load increase of the same amplitude. The secondary control is not activated and not taken into account in the whole study.

In both cases, the hydraulic power in grey is accommodating very quickly the disturbance of the power grid, as there is a sharp power peak. Thermal unit in blue is highly solicited contrary to nuclear power plants.

\subsection{Energy source models}

Source types can be separated into two categories; dispatchable, i.e. conventional power plants such as nuclear, hydraulic or thermal, and non-dispatchable power plants such as wind or solar farms. The thermal and hydraulic regulations available on PowerFactory are taken directly from the IEEE benchmark database. Thus, the hydraulic power unit is modelled by a benchmark governor named HYGOV and the hydraulic power unit model is named IEEEG1 [18]. The modelling of a nuclear power plant required some adaptations described in Figure 1 Section 3.3.1. The variable RES units are represented by the static generator. Section 3.3.2 justifies the modelling used to represent a variable source.

\subsubsection{Building a nuclear model from a thermal unit model}

In general, nuclear governors for primary frequency control do not exist in dynamic simulation software; nuclear energy is then considered as conventional thermal source, since both feature steam turbine cycle [11]. However, current NPPs are limited by ramp of $5 \% \mathrm{Pn} / \mathrm{min}$ [4], which corresponds to the maximum ramp allowed and used for load-following. It was decided to build a model of nuclear regulation starting from a thermal regulation. The purpose of the governor is to restore a steady-state frequency following a grid disturbance without exceeding this maximal power ramp of $5 \% \mathrm{Pn} / \mathrm{min}$. In the study, the maximum ramp achievable by a current nuclear reactor is calculated as the maximum value of the 1-second timeaveraged derivative of the turbine output power in order to avoid non-macroscopic effects.

A thermal or nuclear regulation, which a simplified model is presented in Figure 4 depends mainly on two parameters: the constant of inertia T4 and the droop $s$. [19] details more precisely the building of the nuclear regulation.

NPPs are limited until now by a maximal power ramp of $5 \% \mathrm{Pn} / \mathrm{min}$ for safety reasons but this limit may be challenged in the future. The nuclear regulation follows this limitation thanks to the steam time constant T4. Figure 1 shows that the larger the time constant is, the less quickly the machine reacts and the greater the frequency dip is. It is therefore more delicate to participate in the primary control within the 30 -seconds limit (Tab. 1). Moreover time constant affects directly the power ramp of the machine.

According to [14], the droop linearly relates "the relative quasi-steady-state frequency offset on the network and the relative variation in power output from the generator associated with the action of the primary controller. This ratio without dimension is generally expressed as a percentage". The value is set between 4 and 5\% for nuclear power units. At first order, droop affects the final value of the frequency, as shown in Figure 5. The 5\% value for the droop is kept in the following.

In summary, the time constant $\mathrm{T} 4$ impacts on time response and therefore on the nuclear ramp speed since frequency is directly linked to turbine power, while the droop impacts the amplitude of the frequency dip.

The governor model so-called "IEEEG1" [20] includes the two parameters droop and time constant. It is used as a nuclear regulator in the following with the adequate parameters. 
Table 3. Installed power of the adapted "4-machine" and initial load flow.

\begin{tabular}{llllllll}
\hline & GEN_A1 & GEN_A2 & GEN_B1 & GEN_B2 & Gen_Stat & Load A & Load B \\
\hline Source type & Thermal & Nuclear & Hydraulic & Nuclear & RES farm & - & - \\
Installed capacity & $810 \mathrm{MW}$ & $810 \mathrm{MW}$ & $810 \mathrm{MW}$ & $810 \mathrm{MW}$ & $400 \mathrm{MW}$ & - & - \\
Load flow & $276 \mathrm{MW}^{*}$ & $700 \mathrm{MW}$ & $720 \mathrm{MW}$ & $700 \mathrm{MW}$ & $400 \mathrm{MW}^{* *}$ & $967 \mathrm{MW}$ & $1767 \mathrm{MW}$ \\
\hline
\end{tabular}

* The thermal machine GEN_A1 is the reference machine, its initial power (load flow) depends on the initial power of the added RES source as well as on the load demand. The power of the reference machine, called slack bus, in a load flow program must compensate for the gap between the power demand and the power supply by the other generators (nuclear, hydraulic and RES), taking into account the losses on the network. In reality, several machines adapt their production to avoid a possible overproduction/underproduction on the grid (it is indeed the primary frequency control, which is modelled by a slack bus).

** More explanations on the 400 MW RES power value are given in Section 3.3.2. It can be noticed that the total of load consumption differs from the total production of $59 \mathrm{MW}$ due to the losses in the grid (power lines hundreds kilometres long and presence of transformers).

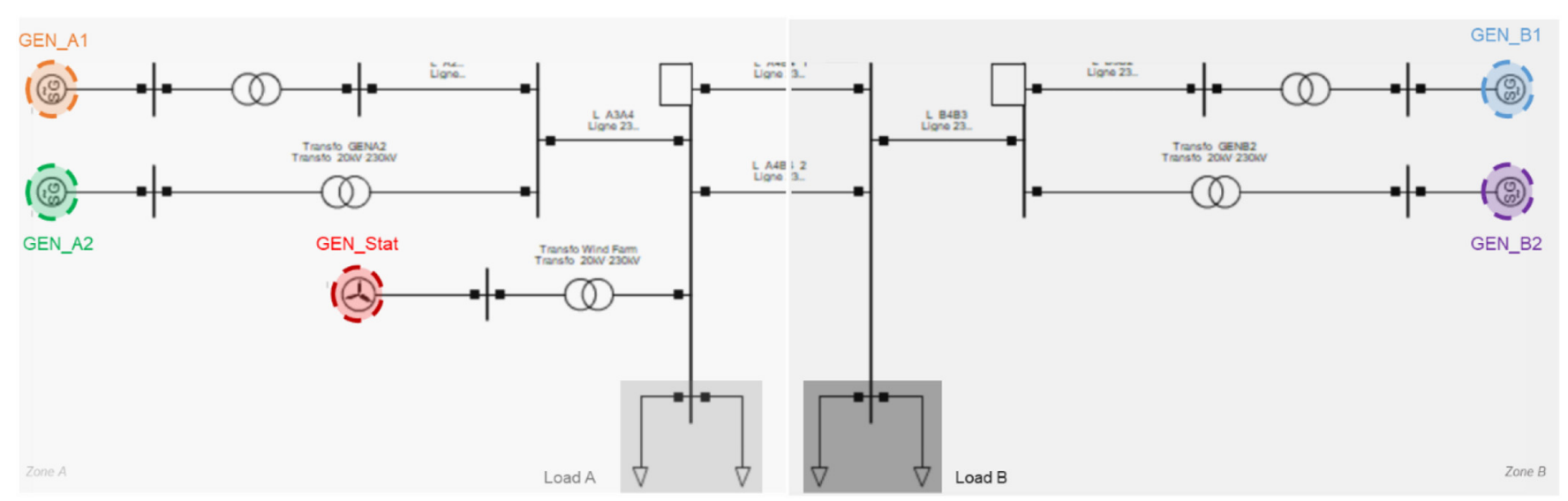

Fig. 2. Kundur's power system with additional RES plant.

\subsubsection{Implementation of variable renewable energy sources on the network}

This section describes the modelling chosen in the study with regards to the insertion of RES such as PV panels and wind turbines. In order to take into account the influence of RES on the network, the three following points need to be addressed:

- electrical conversion and connexion to the grid of variable RES.

- initial conditions of variable RES power.

- "Penalizing" events characterizing the variability of such production.

Wind turbine and PV conversion, and connexion to the grid:

the modelling of a wind farm by using a simple model based for example on a static generator instead of grouping DFIG (Double Fed Induction Generator) turbine farms, as in [19], is possible to avoid long computation times. This way, the local and rapid effects due to the modelling of a synchronous or asynchronous rotating generator with power electronic connection interface do not interfere on the simulation. PowerFactory user's guide recommends the use of such static generators for "wind generators that are connected to the grid using a full-size converter, because the behaviour of the plant (as 'seen' from the grid) is determined by the converter" [11].

Moreover, the static generator is an easy-to-use model to represent any kind of non-rotating generator, such as photovoltaic generators. Indeed, PV generators are connected to the power grid through a power electronic converter.

The RES farm is connected to the same bus as load A, as stated previously (Fig. 2).

\section{Initial conditions:}

It is necessary to determine the proportion at time $t$ of variable source on the network. However, this proportion depends both on the total installed capacity on the network (proportion of RE on the network) and on the loading of RE at the chosen time.

- RES proportions:

the table below gives an example of the proportions of variable RES for the "4-machine" network adapted from two different French mix case (2018 and 2028). 

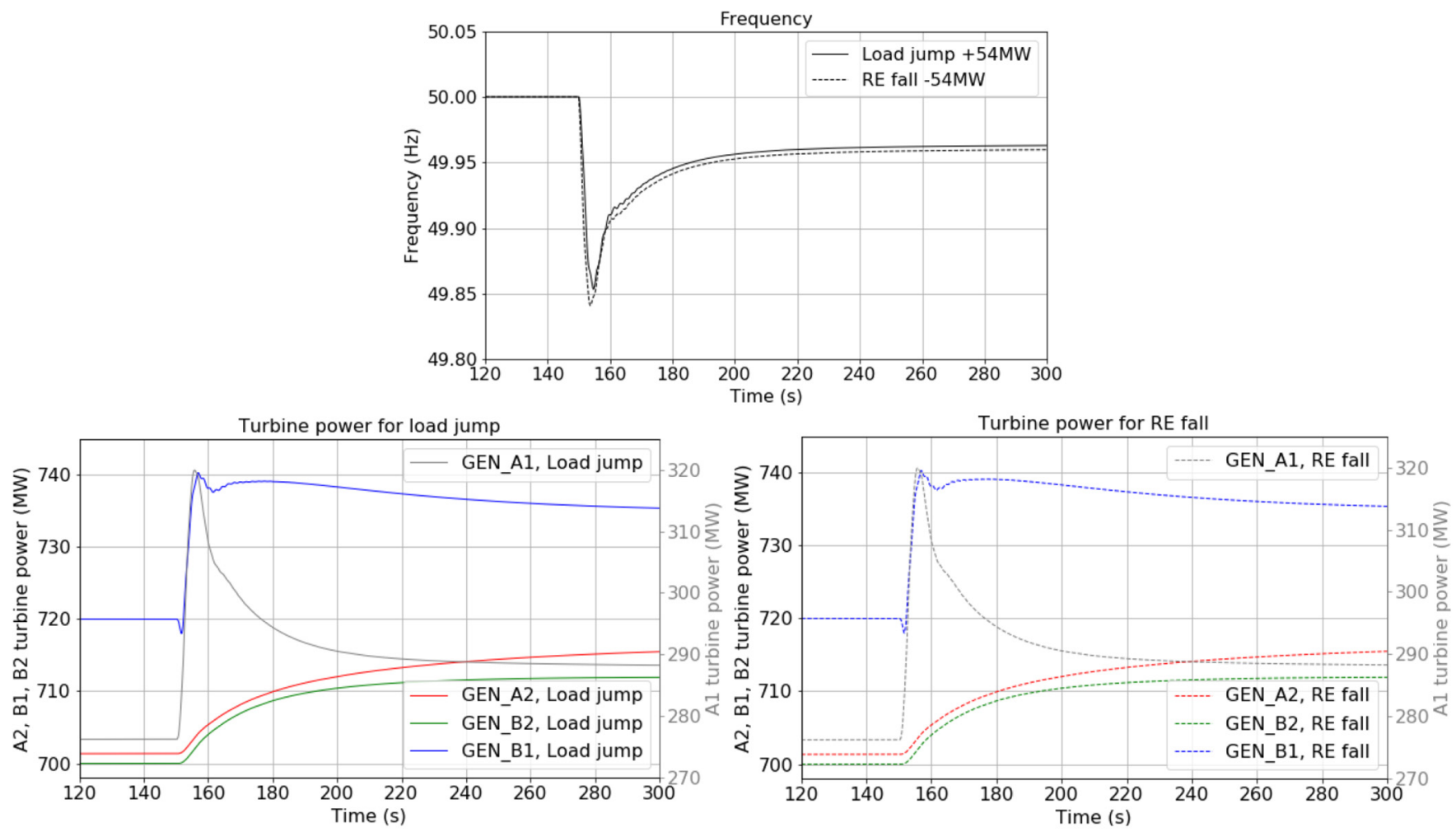

Fig. 3. Frequency (top) and turbine power (bottom) responses for two different 54MW power deviation (load jump in continuous line and RE fall in dotted line).

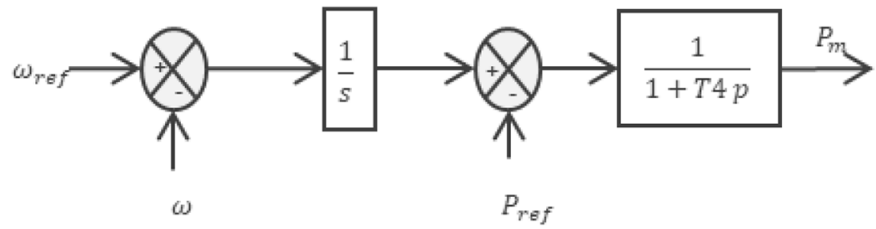

Fig. 4. Simplification of thermal or nuclear regulation.
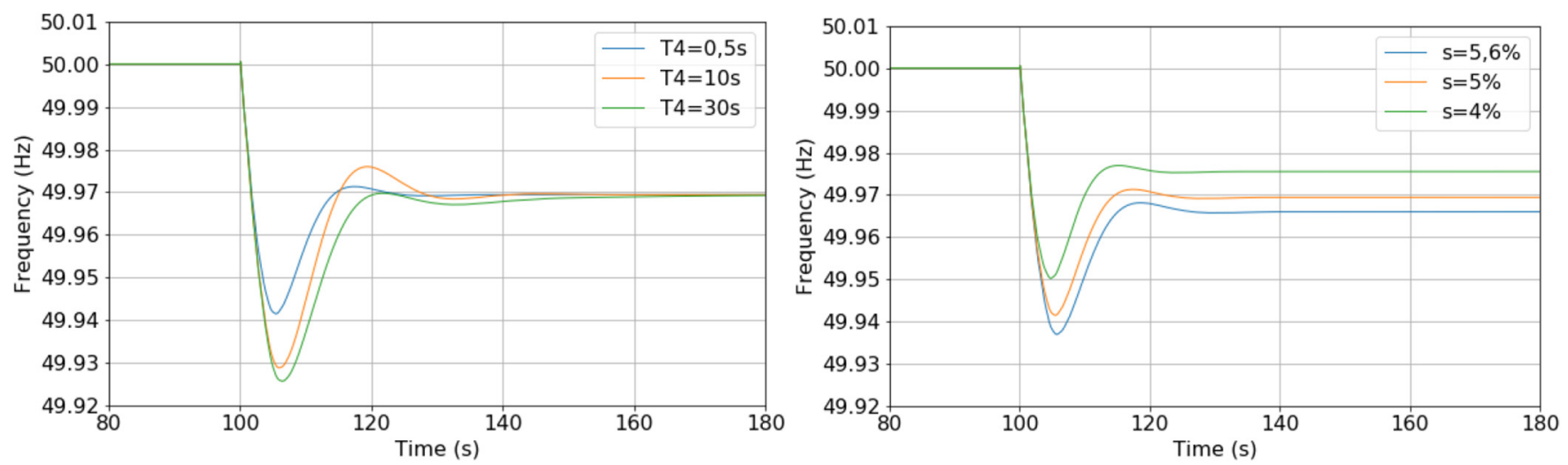

Fig. 5. Frequency response after a $2 \%$ load increase as function of time constant (left) and droop (right). 
Table 4. Projection of installed capacities of wind and solar power plants in 2018 and 2028 for France and the " 4 -groups" equivalent model.

\begin{tabular}{|c|c|c|c|c|c|c|}
\hline & \multicolumn{3}{|c|}{$\begin{array}{l}2018 \text { actual power } \\
\text { mix from the French } \\
\text { TSO [1] }\end{array}$} & \multicolumn{3}{|c|}{$\begin{array}{l}2028 \text { theoretical power } \\
\text { mix according to the French } \\
\text { energy road-map [3] }\end{array}$} \\
\hline & France & 4-groups & Approx. share & France & 4-groups & Approx. share \\
\hline Wind & $15 \mathrm{GW}$ & $450 \mathrm{MW}$ & $11,5 \%$ & $40 \mathrm{GW}$ & $1550 \mathrm{MW}$ & $25 \%$ \\
\hline Solar & $8.5 \mathrm{GW}$ & $250 \mathrm{MW}$ & $6,4 \%$ & $\sim 40 \mathrm{GW}$ & $1550 \mathrm{MW}$ & $25 \%$ \\
\hline Total Wind + Solar & $24 \mathrm{GW}$ & $700 \mathrm{MW}$ & $18 \%$ & $80 \mathrm{GW}$ & 3100 MW & $50 \%$ \\
\hline Total full network & $130 \mathrm{GW}$ & $3940 \mathrm{MW}$ & & $130 \mathrm{GW}$ & $6340 \mathrm{MW}$ & \\
\hline
\end{tabular}

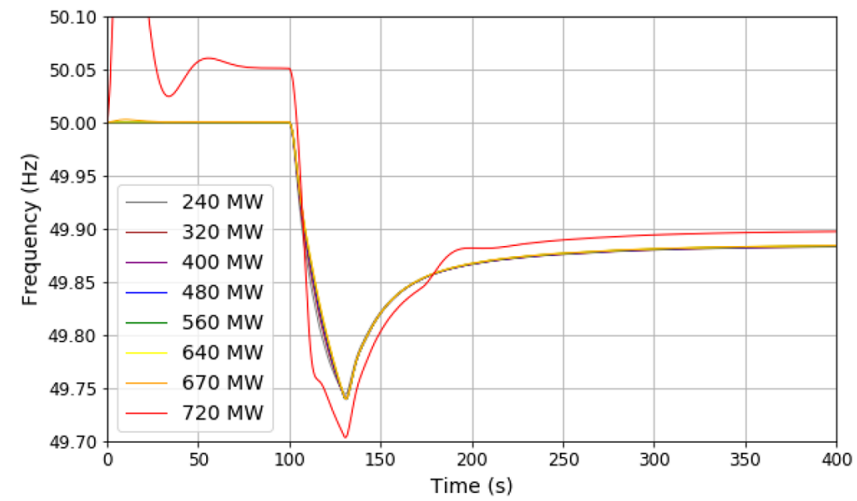

Fig. 6. Frequency response for a $160 \mathrm{MW}$ power drop in $10 \mathrm{sec}$ with different RE initial powers.

According to Table 4, the proportion of added RES is large compared to the total capacity, especially for the 2028 scenario. Such RES proportion in the Kundur's network is not possible. The next paragraph explains how to deal with this limit and the modelling of RES power.

- RES output power:

the original "4-groups" network was initially designed to model inter-area oscillations, which is the reason why as soon as the load flow deviates from the nominal operating point (load flow), frequency instabilities are quickly observed. Thus, simulations of the study perform close to the operating point, and the RES output power variation from initial value is then limited.

The maximum wind power production in France was achieved by using $80.3 \%$ of the wind power production capacity. The same applies to solar energy, with a maximum load factor of $75 \%$ [1].

However, initial RES powers do not influence the frequency response after the same RES power fall, as shown in Figure 6, which describes the response to a 160 MW drop of the output power of the RES farm.

Moreover, according to Figure 6, the RES load cannot exceed 670 MW of RES power, otherwise the network becomes unstable (red curve) for a $160 \mathrm{MW}$ disturbance. This is because the system has a small number of machines and the total capacity of $810 \mathrm{MW}$ of the reference machine (GEN_A1), cannot be exceeded to compensate for a possible disturbance.

The frequency response depends exclusively on the total power gap (160 MW in the example above) and the equivalent droop of the units participating in the primary control. The initial power of the RES units has no influence on the final frequency deviation according to equation (2) below [17].

$$
\Delta f=\frac{f_{0} \Delta P}{\sum_{i=1}^{m} \frac{P_{n i}}{s_{i}}}
$$

with $\Delta P$ Total power deviation; $P_{n_{i}}$ rated power of machine $i ; s_{i}$ droop of machine $i ; f_{0}$ rated frequency of $50 \mathrm{~Hz} ; m$ number of machines.

Therefore, the RES output power will set under 650 MW in the next sections. RES power output capacity is considered having no influence on the grid stability as long as it remains below the maximum swing machine capacity in this model.

\section{Penalizing RES event}

The variability of RES and the corresponding parameters must be defined in order to characterize the penalizing event associated to the variable RES. 


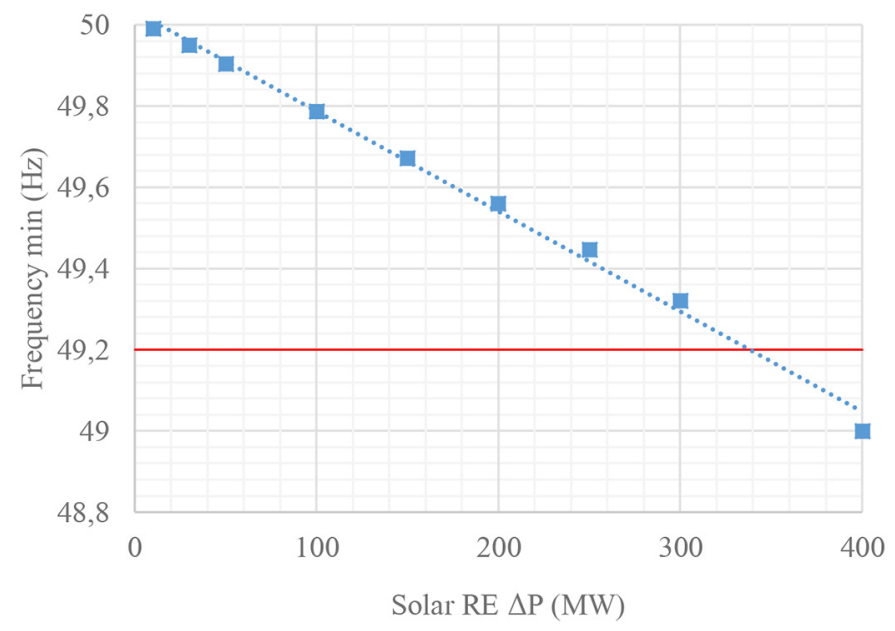

Fig. 7. Frequency dip as a function of the variable solar RE magnitude drop with thermal group.

The energy supplied by the RES production is, among other things, linked to climatic conditions (solar wind): a fine characterization of the variability is therefore necessary. Even if efforts are made concerning forecasting, today, the variable energies (wind and solar) are not controllable. The objective is to identify a single event that would disturb the system due to intermittency, either the most penalizing one (e.g. sudden power drop), or an average event at a regional scale. The objective being to model a flexible reactor for sudden and abrupt changes occurring on the network, it is preferable to find the most penalizing event for a short time and intend to correct it with the primary control. In order to characterize this event, the most relevant parameters remain the power variation $\Delta P$ within a period of time $\Delta t$, which means knowing the variation $\operatorname{ramp} \% P /$ time.

However, data on regional or national geographical scales of variable RES generation are not available in open source for short times (less than one minute) as the study focuses on primary frequency control.

The data accessible for the corresponding time scale are those of a single wind turbine or solar panel - or those of a solar or wind farm at best. After a non-exhaustive bibliographical analysis [21,22], the penalizing event values selected for the penetration of variable RES at the French national scale are:

- Solar: $\pm 60 \% \mathrm{Pn} / \mathrm{min}$ or $1 \% \mathrm{Pn}$ in $1 \mathrm{~s}$

- Wind: $\pm 1 \% \mathrm{Pn} / \mathrm{min}$ or $1 \% \mathrm{Pn}$ in $1 \mathrm{~min}$.

Considering the selected values mentioned above, the wind variation will be overlooked in favour of the solar variation on first examination. Indeed, the solar ramp seems restrictive for wind source than PV power. This ramp of $1 \% \mathrm{Pn} / \mathrm{min}$ is manageable with current nuclear, but technology could be penalizing when accumulated with other disturbances. Thus, only solar disturbances are taken into account in the subsequent sections.
This study is not intended to be exhaustive but gives order of magnitudes of a realistic recorded event. These first values are sufficient for this approach which interest lies in highlighting trends. Further studies could allow for the improvement of the results of this paper.

In addition, it is assumed in this study that increasing the variable RES penetration is similar to increasing the magnitude of RES power variation as installed capacity of RES has no influence on the power grid.

\subsection{Simulation of the current case with the considered adjustments}

The system has been adapted to represent an equivalent French network with the addition of a RES source. The RES power plant is initially set to generate $500 \mathrm{MW}$. However, this initial value has no impact, as the installed capacity does not influence transients (Sect. 3.3.2). Then, a solar RES drop of $10 \% \mathrm{Pn}$ in 10 s occurs. This RES fall represents approximately a penalizing event of the current mix as explained in Section 3.3.2. The frequency follows the decrease of RES production as shown in the top of Figure 8, until the primary reserve is fully activated. However, it remains in the acceptable range of $\pm 0.8 \mathrm{~Hz}$ transiently and $\pm 0.2 \mathrm{~Hz}$ in steady state operation, which is consistent with the low RES power variation. The disturbance is distributed over all the machines, even though the two nuclear units (red and yellow curves) are less stressed compared to the thermal and hydraulic machines. The response of the nuclear units verifies the maximal $5 \% \mathrm{Pn} /$ min ramp criterion.

This representation of a realistic RES drop event on an existing power system with a varied mix of thermal, hydraulic and nuclear power is therefore consistent, given that the existing current power grid is fully capable of accommodating such a disturbance.

\section{Stability study with a "stressed" power system}

This section stresses the power system described before by progressively inserting intermittent renewable energy installed capacity all the while reducting thermal resources in order to fulfil the energetic scenario that France considers $[2,3]$. The influence on stability of the two effects is studied separately and then, the phenomena are combined.

\subsection{Impact of the magnitude drop of the variable RES disturbance}

Current policies aim at the increase of wind and solar production up to a capacity representing half of the French installed base in a decade. Since the installed capacity has no influence on the frequency response (Sect. 3.3.2), the initial power in RES remains set at 500 MW. The proportion of variable RES on the total mix varies from $5 \%$ to $60 \%$, which corresponds, according to Section 3.3.2, to a range of magnitude of $10 \mathrm{MW}$ to $400 \mathrm{MW}$ (400 MW corresponds to about $10 \%$ of the total installed capacity). 

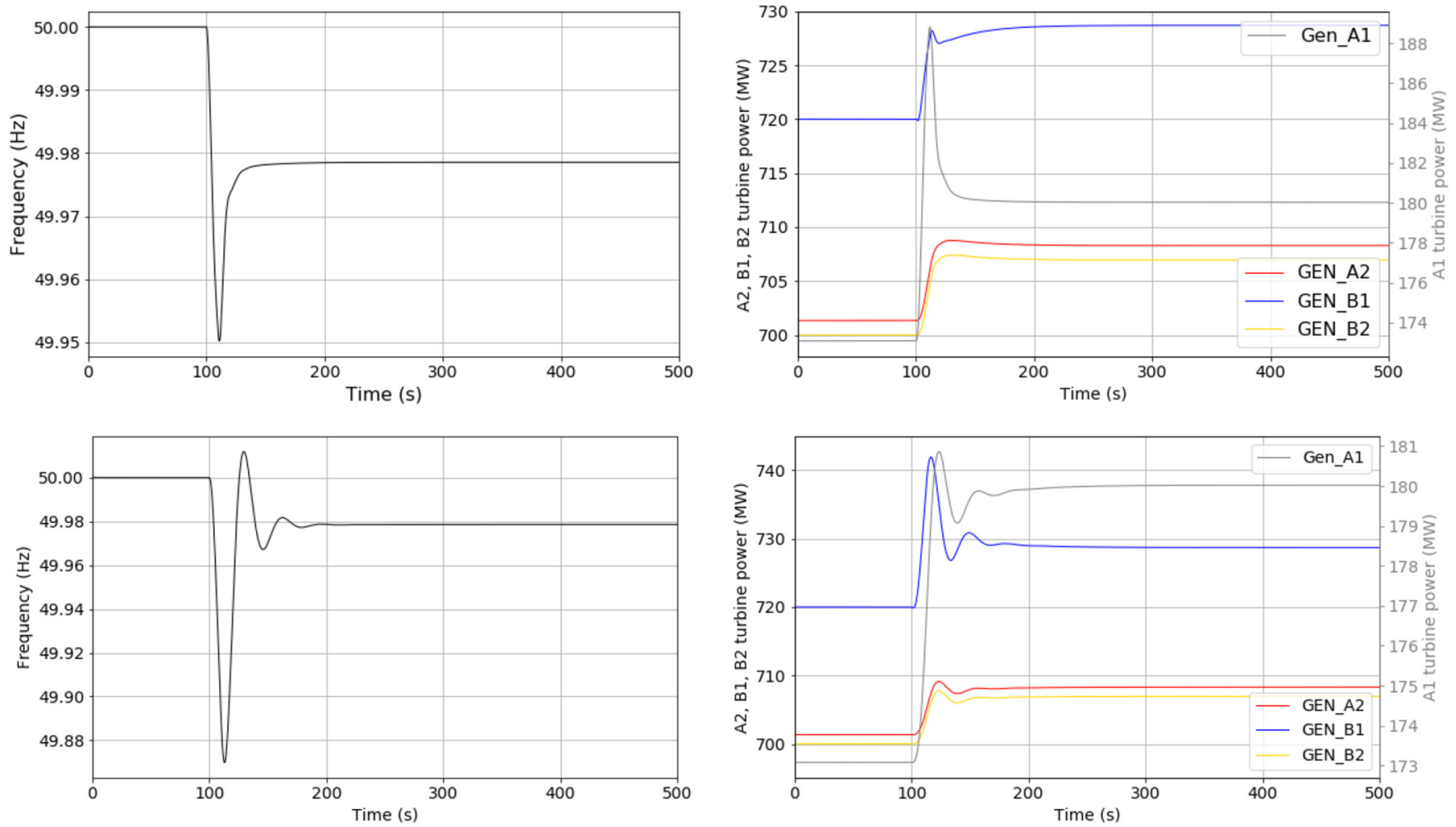

Fig. 8. Frequency response (left) and turbine powers (right) after a $30 \mathrm{MW}$ RE power fall with thermal group (top) and without thermal group (bottom).

According to the blue curve of Figure 7, the higher the variable solar $\mathrm{RE}$ power drop, the higher the minimal frequency. In addition, the frequency drop even exceeds the permissible stability limit of $49.2 \mathrm{~Hz}$ (red line in the figure below) for more than $340 \mathrm{MW}$ of solar RES power drop. This graph represents the margin (or not) to the stability as a function of the solar RES magnitude drop.

\subsection{Impact of the removal of thermal power}

The impact of the replacement of thermal generating units (gas, diesel, coal) by nuclear power plants on the stability of the is then further explored. For example, the 4-machine network is now composed of three nuclear machines (instead of two initially), one hydraulic machine and one RES power plant producing $500 \mathrm{MW}$ initially. A $30 \mathrm{MW}$ RES production drop is considered like in the case presented in Section 3.4. Although the frequency criteria are fulfilled, the network seems more unstable in the case of without thermal unit, according to Figure 8, as oscillations occur on the frequency response in the case of no thermal unit (bottom of the figure). A second excess beyond $50 \mathrm{~Hz}$ occurs but remains in the right place of operation. Reducing the share of fossil fuels leads to a lower dip frequency. The oscillations, which appear, as expected, at the bottom of Figure 8 may be reduced by adjusting, among other things, governor regulation parameters.
Furthermore, it is not possible to gradually reduce the share of thermal power on this network. The unit operates either with the thermal control or the thermal control is replaced with another one such as nuclear or hydraulic. There is no intermediate case. In the subsequent section, the thermal unit is fully replaced by a nuclear model.

\subsection{Alternative scenario with more variable RES and less thermal generation}

Both previous studied scenario are combined (Sects. 4.1 and 4.2), which means that the $\mathrm{CO}_{2}$ emissions are reduced by simultaneously removing fossil fuel power plants (replaced by nuclear power plant) and by increasing RES penetration. It results in the orange curve of Figure 9 added to the blue curve already presented in Figure 7.

Figure 9 illustrates the fact that current nuclear power limited by $5 \% \mathrm{Pn} / \mathrm{min}$ is currently sufficient to ensure stability but will not be sufficient in its current state according to the paths taken to reduce fossil fuel production. On the one hand, there is a different inclination between the blue curve (with thermal) and the orange curve (without thermal) because the rapid response capacities are lower in the case without a thermal machine; only the hydraulic machine is able to respond quickly to the disturbance. There is also a break in the slope after $125 \mathrm{MW}$ RES drop on the orange curve (without thermal) because 


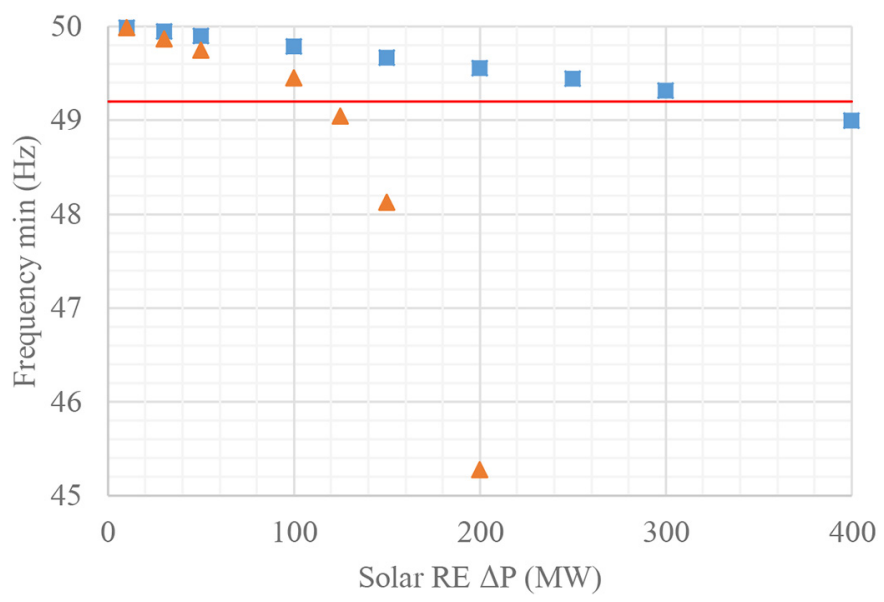

Fig. 9. Frequency dip as a function of the variable solar RE magnitude drop with and without thermal support.

Table 5. Summary of the studied cases.

\begin{tabular}{llll}
\hline Section & Scenario & Stability & Stability remarks \\
\hline 3 & Reference case & Yes & - \\
4.1 & More variable RE power & Yes & - \\
4.2 & Removal of thermal unit & Yes & Frequency oscillations appear \\
4.3 & $\uparrow$ variable RE power $+\downarrow$ thermal power & No & Full primary reserve are deployed \\
\hline
\end{tabular}

the primary reserves are no longer sufficient to return above the admissibility level (red line). The only flexibility lever: hydraulic unit is saturated (see Sect. 5.1).

On the other hand, the admissibility limit for stability is exceeded for a lower drop of solar RES power, as low as $125 \mathrm{MW}$.

Table 5 summarizes the studied cases and their associated stability.

For the last case, a scenario strongly envisaged in the future [3], additional flexibility levers are therefore necessary. It is then proposed to restore stability using flexible nuclear power generators in the next section.

\section{Influence of flexible nuclear power to restore stability}

The following section focuses on the cases that do not check the stability limit set at $49.2 \mathrm{~Hz},-$ transients below the red line in Figure 7. The idea of this part is to determine the capacity of modumation for nuclear power plants necessary to restore stability - and thus return to the normal operating band - if nuclear power were to be more flexible and the only lever of flexibility except for one hydraulic unit. First of all, a non stable transient is studied in order to observe the instability caused by the high variable RES penetration with no flexible machine. Then, the nuclear ramp is adapted in order to restore stability. The last subsection gives the flexible nuclear ramp as a function of the variable solar RES power drop.

\subsection{Behavior of a non-stable transient}

Figure 10 considers the first case without a thermal unit that is not within the range of admissibility in Figure 7. This scenario represents approximately the forecast envisaged for 2028, i.e. total thermal reduction and 50\% of RES in the total installed capacity. Incidence of wind power variability is, as supposed in Section 3.3.2, neglected. In addition, there are three nuclear units and one hydraulic unit.

In this case, shown in the figure below, the stability indicators are not satisfied; the frequency (left) no longer respects its transient operating band. The frequency drop is high and exceeds the limit value of $49.2 \mathrm{~Hz}$ transiently. However, the frequency stabilizes within the acceptable frequency range of $49.8 \mathrm{H} z$. The three nuclear machines are slower and do not allow the frequency drop to be limited because these units are unable to release their primary reserve quickly enough. The turbine power stage of the hydraulic unit in blue shows that the machine has reached its maximum primary reserve. This is the reason why the orange curve of Figure 7 shows a slope break. This means also that it is no longer normal operating conditions 

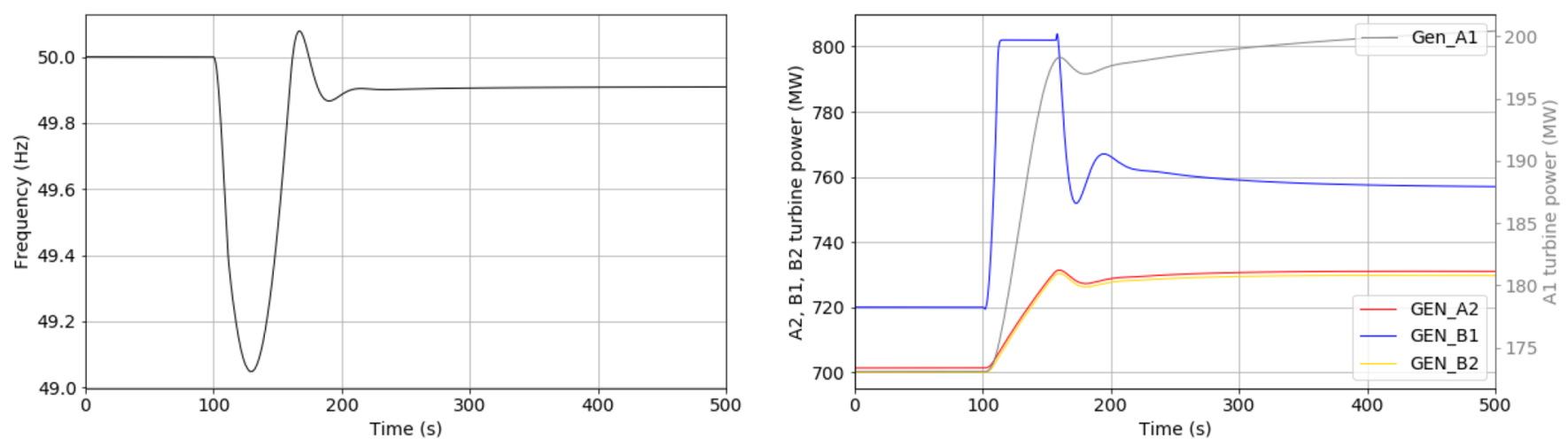

Fig. 10. Frequency transient for $125 \mathrm{MW}$ solar power drop with 3 NPPs and 1 hydraulic power plant.

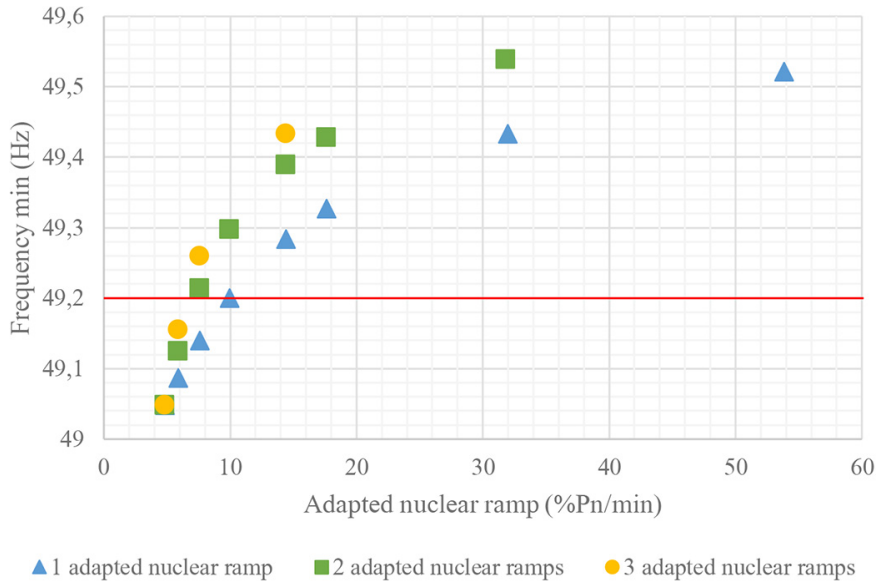

Fig. 11. Dip frequency as a function of the adapted nuclear ramp for $125 \mathrm{MW}$ solar RE power drop.

(Sect. 2.4) and the system becomes unstable. Indeed, the thermal power plant is no longer involved, substituted by the nuclear power.

This specific mix cannot support such a sudden variation in generation due to the high proportion of RES and the lack of flexible unit. The transient represented in the figure is not conceivable. More flexible resources, such as those previously provided by thermal units, are therefore required in order to bring back the frequency to its operating range and to restore the power system stability.

\subsection{Adaptation of the nuclear ramp}

The two figures below show the nuclear ramp adapted for two fixed solar RES power drops, initially not fulfilling the stability condition, as a function of the frequency dip.

125 MW solar RE power drop:

with a single flexible nuclear machine, a minimum ramp of $10 \% \mathrm{Pn} / \mathrm{min}$ would be required to restore stability for a solar RES power drop of $125 \mathrm{MW}$. If the ramp is further improved (higher), the margin of stability is thus more consequent. With the addition of a flexible nuclear machine or even two, the minimum ramp required is less restrictive. The minimal ramp is $7 \% \mathrm{Pn} / \mathrm{min}$ for two nuclear units and $6.5 \% \mathrm{Pn} / \mathrm{min}$ for three nuclear units.

$150 \mathrm{MW}$ solar RE power drop:

a second non-stable point is studied in order to deviate from the stability limit and return above the frequency limit of $49.2 \mathrm{~Hz}$. Same remarks as for the previous figure are observed. (1) If the nuclear ramp is further improved, the margin of stability is thus more consequent. (2) With additional flexible nuclear machines, the minimum ramp required is less restrictive. But, in this case, the adapted nuclear ramp must be much higher in order to restore stability. A minimal adapted ramp of $32 \% \mathrm{Pn} / \mathrm{min}$ is required for one flexible machine, and between $15 \% \mathrm{Pn} / \mathrm{min}$ and $17 \% \mathrm{Pn} / \mathrm{min}$ for two to three adapted nuclear ramps.

\subsection{Flexible nuclear ramp}

The initial objective of the approach is to connect directly the minimum ramp of flexible nuclear to restore stability according to the solar RES power drop. Figure 13 shows the flexible ramp for a variable solar RES drop. The flexible ramp is calculated in such a way that the frequency verifies the frequency limit criterion at $49.2 \mathrm{~Hz}$. 


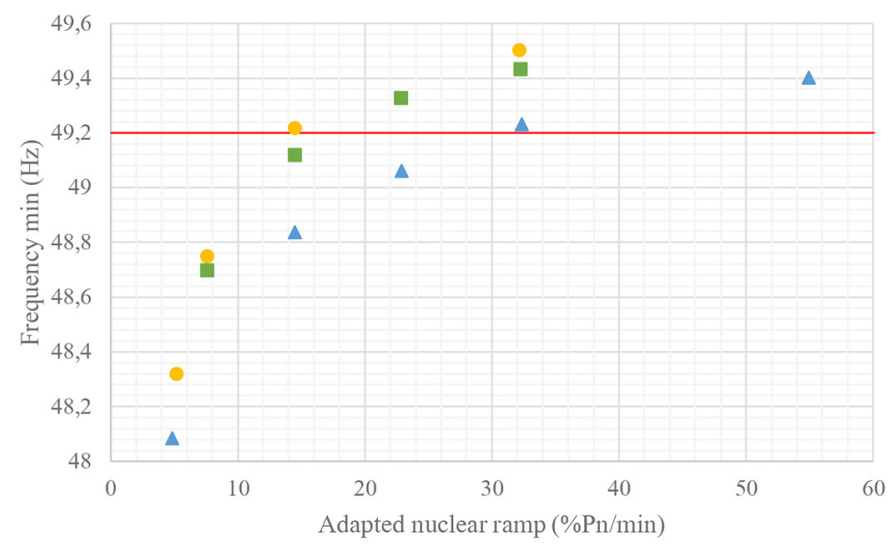

$\Delta 1$ adapted nuclear ramp $\quad \square 2$ adapted nuclear ramps $\quad 3$ adapated nuclear ramps

Fig. 12. Dip frequency as a function of the adapted nuclear ramp for $150 \mathrm{MW}$ solar RE power drop.

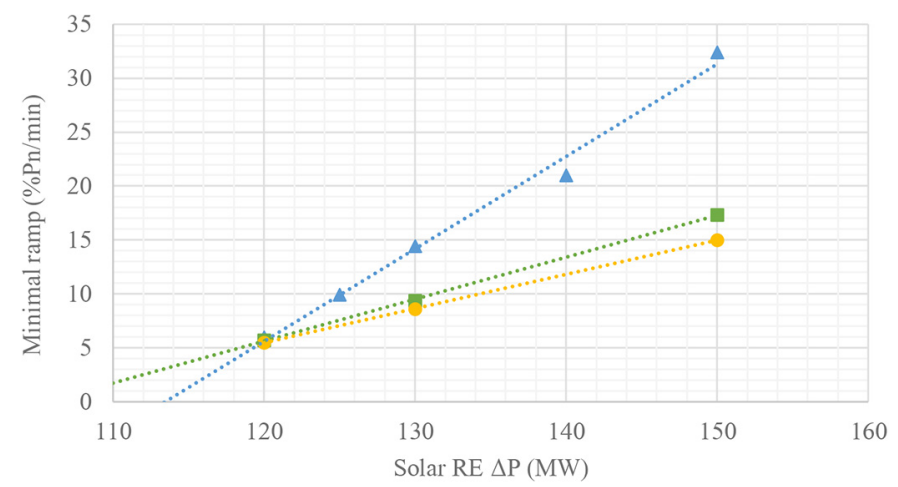

$\Delta 1$ flexible nuclear $\quad$ 2 flexible nuclear 3 flexible nuclear

Fig. 13. Flexible nuclear ramp as function of the solar RE magnitude drop.

The higher the RES insertion is, the more the flexible ramp must compensate and therefore increase. For example, for a constrained case of $140 \mathrm{MW}$ of solar RES in installed capacity, a $22 \% \mathrm{Pn} / \mathrm{min}$ nuclear ramp is required for a single flexible machine. If other flexible nuclear machines are added, the flexibility of the machine may be less, i.e. about $13 \% \mathrm{Pn} / \mathrm{min}$.

The addition of a third flexible machine in the conditions of the studied network is not necessarily relevant, because the third machine does not provide additional stability; indeed, yellow and green curves in Figure 11 and Figure 12 are very close.

\section{Conclusion and perspectives}

The decarbonisation of the electrical system is one of the major challenges of the upcoming decades. To this end, current policies are moving towards the massive penetration of variable renewable energy sources and the elimination of fossil fuel units (coal, gas, and diesel) due to their high $\mathrm{CO}_{2}$ emissions. Disturbances on the electric network may therefore soon increase with the progressive insertion of these variable RES and the decrease of flexible power plants. For this reason, some carbon-free dispatchable generation units (controllable by the grid operator) are still necessary for the good operation of the electric grid, in order to ensure a balance between electricity generation and consumption at all times. These flexibility capacities are necessary to provide the transmission system operator with fast power reserves. This balance is mainly ensured by the participation of rotating machine units in frequency control, including primary frequency control. Today, flexible power plants such as thermal and hydraulic units may provide a power ramp of more than $50 \% \mathrm{Pn} / \mathrm{min}$ for hydraulic, and $20 \% \mathrm{Pn} / \mathrm{min}$ for conventional thermal plants contrary to nuclear units which are limited to $5 \%$ $\mathrm{Pn} / \mathrm{min}$.

This paper suggested an approach to highlight these new challenges for the network and to quantify the impact of these variable RES on the stability of the system. The proposed approach provides then a preliminary assessment of the main requirements for nuclear flexible reactors to accommodate highly variable power systems. For this purpose, it was necessary to build a study framework representative enough of the current French transmission power system, also taking into account future considerations and solicitations (RES insertion). This network has also been subject to adjustments such as the elaboration of a nuclear control model that verifies the $5 \% \mathrm{Pn} / \mathrm{min}$ limit and, on the other hand, the implementation of a so-called "penalizing" but realistic event due to a fluctuation in variable RES generation. Finally, this network model made it possible to deduce, in the study framework, the flexibility requirement for nuclear power according to different cases of insertion of solar RES. This study also highlighted the essential role of having a flexibility lever currently provided by thermal and hydraulic power. As soon as the thermal machine was removed, stability was no longer achieved. Transients became more and more critical with the strong insertion of the RES. The addition of one or two flexible nuclear power units allows a return to stability. However, the third flexible NPP does not provide substantial stability benefits. This paper has therefore highlighted an approach that allow linking a potential flexible nuclear power plant to the sizing of a electric network.

However, the model used in the paper called Kundur's "2-area 4-machine" does have limitations such as the limited integration of variable RES in terms of installed capacity, the inherent instability of the Kundur network which has to run close to its nominal operating point or the low nominal capacity of the electric network and the number of machines.

In further works, the use of a more complex/realistic network such as the 39-bus [23,24], which is a simplified scheme from a real north-american grid, should remove these constraints. A robustness study will make possible to consolidate the preliminary results using the 4-machine network and resulting in the approach described throughout the paper. In perspective, it is also envisaged to implement the flexible nuclear design in a network in order to verify its suitability for the needs of the network. 


\section{Author contribution statement}

This paper is part of the research work carried out in the framework of the thesis of A-L. Mazauric. P. Sciora, J-B. Droin and V. Pascal from CEA have supervised the work, as well as N. Hadjsaid, Y. Bésanger from G2elab. They assisted in the development of the method and in the implementation of the models. N. Guyonneau, from CEA, provided improvements on the consideration of the RE source. QT. Tran, from CEA, contributed some elements on the characterization of the RE variability part. All authors contributed to the bibliography part, discussed the results and contributed to the final manuscript.

\section{References}

1. RTE, Bilan électrique 2018, 2018

2. Journal Officiel de la République Française, Loi n²015-992 du 17 août 2015 relative à la transition énergétique pour la croissance verte, 2015

3. Ministère de la Transition Ecologique et Solidaire, "French strategy for energy and climate: multi annual energy plan, 2019-2023, 2024-2028," 2019

4. IAEA, Non-baseload Operation in Nuclear Power Plants: Load Following and Frequency Control Modes of Flexible Operation, IAEA Nuclear Energy Series, 2018

5. T.E. Baker et al., Optimal sizing of flexible nuclear hybrid energy system components considering wind volatility, Appl. Energy 212, 498-508 (2018)

6. C. Forsberg, Electricity production using nuclear reactors with topping cycles to compete with low-price natural gas and subsidized renewables, in Proceedings of the 2016 International Congress on Advances in Nuclear Power Plants (ICAPP 2016), 2016

7. G. Locatelli et al., Cogeneration: an option to facilitate load following in small modular reactors, Progr. Nucl. Energy 97, 153-161 (2017)

8. E. Redfoot, R.A. Borelli, Analysis of nuclear renewable hybrid energy systems modeling and nuclear fuel cycle simulators, Nucl. Technol. 204, 249-259 (2018)

9. Entso-e, Operation Handbook - Glossary, UCTE, 2004
10. RTE, Memento de la sûreté du système électrique, 2004

11. DIgSILENT, PowerFactory - User manual, 2018

12. C. Bruynooghe et al., Load-following operation mode at nuclear power plants (NPPs) and incidence on operation and maintenance (O\&M) costs. Compatibility with wind power variability, JRC Scientific and Technical Reports $n^{\circ}$. EUR 24583 EN, JRC European Commission, 2010

13. RTE, Documentation Technique de référence, Chapitre 4 Contribution des utilisateurs aux performances du RPT, Article 4.1-Réglage Fréquence/Puissance, 2011

14. ENTSO-e, UCTE Operation Handbook (Appendix 1: Loadfrequency control and performance), 2004

15. P. Kundur, Power System Stability and Control (McGrawHill, New-York, 1994)

16. J-C. Passelergue, Interactions des dispositifs FACTS dans les grands réseaux électriques (Institut National Polytechnique de Grenoble, 1998)

17. S. Ammari, Interaction des dispositifs FACTS avec les charges dynamiques dans les réseaux de transport et d'interconnexion (Institut National Polytechnique de Grenoble, 2000)

18. IEEE Committee Report, Dynamic models for steam and hydro turbines in power system studies, IEEE Trans. Power Apparat. Syst. PAS-92 6, 1904-1915 (1973)

19. A-L. Mazauric et al., Toward the specifications of a "flexible" nuclear reactor: Approach through power system, in ICAPP proceedings (2021, under publication)

20. https://www.powerworld.com/WebHelp/Content/Transi entModels_PDF/Generator/Governor/Governor\% 20IEEEG1\%20and\%20IEEEG1_GE.pdf, consulted on $2019 / 10 / 21$

21. V. Courtecuisse, Supervision d'une centrale multisources à base d'éoliennes et de stockage d'énergie connectée au réseau électrique, École Nationale Supérieure d'Arts et Métiers, 2008

22. RTE, https://www.rte-france.com/eco2mix

23. T. Athay et al., A practical method for the direct analysis of transient stability, IEEE Trans. Power Apparat. Syst. PAS98, 573-584 (1979)

24. M.A. Pai, Energy function analysis for power system stability, 1989

Cite this article as: Anne-Laure Mazauric, Pierre Sciora, Vincent Pascal, Jean-Baptiste Droin, Yvon Bésanger, Nouredine Hadjsaïd, Quoc Tuan Tran, Nicolas Guyonneau, Simplified approach to determine the requirements of a "flexible nuclear reactor" in power system with high insertion of variable renewable energy sources, EPJ Nuclear Sci. Technol. 8, 5 (2022) 